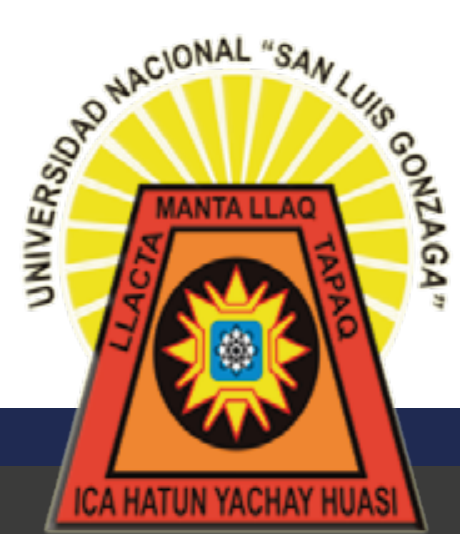

p-ISSN 2223-2893

e-ISSN 2225-6989

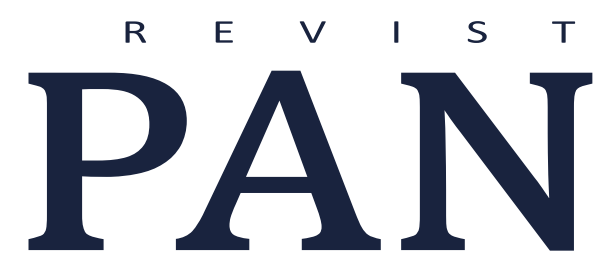

UNIVERSIDAD NACIONAL SAN LUIS GONZAGA. ICA, PERÚ

FACULTAD DE MEDICINA HUMANA "DANIEL ALCIDES CARRIÓN"

ARTÍCULO ORIGINAL:

VOLUMEN 10 NÚMERO 2 PUBLICACION CUATRIMESTRAL MAYO - AGOSTO

2021

\title{
ANOMALÍAS DENTOFACIALES SEGÚN EL SEXO, EN ESTUDIANTES DE ODONTOLOGÍA DE UNA UNIVERSIDAD PÚBLICA DE ICA-PERÚ.
}

DENTOFACIAL ANOMALIES ACCORDING TO SEX IN DENTAL STUDENTS OF A PUBLIC UNIVERSITY OF ICA-PERU.

\section{AUTORES:}

JOSÉ BENJAMÍN MAGALLANES REYES

CLEMENTE CANDELARIO LARA HUALLCCA

BLADIMIR DOMINGO BECERRA CANALES

INDEXADA EN:

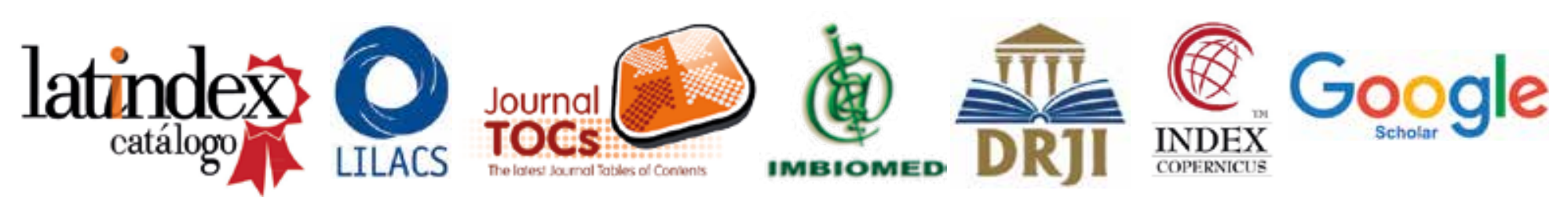

REVISTAS.UNICA.EDU.PE 


\section{ANOMALÍAS DENTOFACIALES SEgÚN EL SEXO, EN ESTUDIANTES DE ODONTOLOGÍA DE UNA UNIVERSIDAD PÚBLICA DE ICA-PERÚ. DENTOFACIAL ANOMALIES ACCORDING TO SEX IN DENTAL STUDENTS OF A PUBLIC UNIVERSITY OF ICA-PERU.}

Magallanes-Reyes José Benjamín ${ }^{1, a, b}$, Lara-Huallcca Clemente Candelario ${ }^{1, a, c}$, Becerra Canales Bladimir Domingo ${ }^{1, a, d}$.

1. Universidad Nacional San Luis Gonzaga. Facultad de Odontología. Ica, Perú

a. Doctor en Salud Publica b. https://orcid.org/0000-0003-2013-7189

c. https://orcid.org/0000-0002-5544-6144

d. https://orcid.org/0000-0002-2234-2189

DOI: $10.35563 /$ rmp.v10i2.426

Correspondencia:

José Benjamín Magallanes

Reyes

Dirección:

Correo electrónico:

jose.magallanes@unica.edu.pe Celular: 956063888

Contribuciones de autoría: JBMR: participó en la concepción de la idea de investigación, diseño del estudio, recolección de datos y aprobación final del manuscrito.

CCLH: participó en el diseño del estudio, recolección de datos y aprobación final del manuscrito.

BBC: participó en el análisis, redacción y revisión del manuscrito.

Conflicto de intereses: no existen conflictos de intereses del autor o autores de orden económico, institucional, laboral o personal.

Financiamiento:

Autofinanciado.

\section{RESUMEN}

Objetivo: Comparar las anomalías dentofaciales según el sexo, en estudiantes de odontología de una universidad pública de Ica-Perú. Materiales y métodos: Estudio de tipo observacional, transversal y descriptivo, realizado en la Universidad Nacional San Luis Gonzaga; durante el año 2019, participaron 60 estudiantes de odontología. Se emplearon modelos ortodóncicos de estudio y se evaluó la severidad de las maloclusiones con el Índice Estético Dental (DAI). Se describieron las variables y evaluaron diferencias mediante el Chi-cuadrado. Resultados: Las anomalías dentofaciales más frecuentes fueron el apiñamiento dental (83,3\%); máxima irregularidad mandibular (73,3\%); máxima irregularidad maxilar (65\%); relación molar en sentido anteroposterior (46,7\%) y superposición maxilar (33,3\%). La máxima irregularidad mandibular (38,3\%) y maxilar (35\%) fue mayor en el sexo femenino; así como, la relación molar en sentido anteroposterior (28,3\%); la superposición maxilar (23,3\%), fue mayor en el sexo masculino. Conclusiones: Se demostró que no existen diferencias estadísticas entre la presencia de anomalías dentofaciales según el sexo de los estudiantes de odontología.

Palabras clave: Deformidades dentofaciales; maloclusion; odontologia.

\section{ABSTRACT}

Objective: To compare dentofacial anomalies according to sex in dental students at a public university in Ica-Peru. Material and methods: Observational, cross-sectional and descriptive study, carried out at the Universidad Nacional San Luis Gonzaga; during the year 2019, 60 dental students participated. Orthodontic study models were used and the severity of the malocclusions was evaluated with the Dental Aesthetic Index (DAI). Variables were described and differences were evaluated using Chi-square. Results: The most frequent dentofacial anomalies were dental crowding (83.3\%); maximum mandibular irregularity (73.3\%); maximum maxillary irregularity (65\%); anteroposterior molar relation (46.7\%) and maxillary overlapping (33.3\%). The maximum mandibular irregularity (38.3\%) and maxillary irregularity (35\%) was higher in the female sex; as well as molar relation in anteroposterior direction (28.3\%); maxillary overlapping (23.3\%), was higher in the male sex. Conclusions: It was shown that there are no statistical differences between the presence of dentofacial anomalies according to the sex of the dental students.

Key words: Dentofacial deformities; malocclusion; dentistry.

\section{Cómo citar:}

Magallanes Reyes J, Lara Huallcca C, Becerra-Canales B. Anomalías dentofaciales según el sexo, en estudiantes de odontología de una universidad pública de Ica-Perú. Rev méd panacea 2021;10(2):70-74. DOI:

10.35563/rmp.v10i2.426 


\section{INTRODUCCIÓN}

Las anomalías dentofaciales o maloclusiones, son alteraciones no patológicas del crecimiento y desarrollo, causan alteraciones en la dentición, la articulación temporomandibular (ATM), las estructuras craneofaciales, neuromusculares y otros componentes del sistema estomatognàtico; que ocasiona un problema funcional; ocupan un lugar relevante dentro de las alteraciones bucodentales en la población. Pueden producir alteraciones osteomusculares, acompañadas de una sintomatología molesta para el paciente; tienen repercusión en la estética y las funciones propias del sistema estomatognático, como la masticación, deglución, respiración y fonación. (1)

Las maloclusiones constituyen el $70 \%$ de los trastornos bucales y representan un problema de salud pública importante. Estas alteraciones según la Organización Mundial de la Salud (OMS), ocupan el tercer lugar, por tanto su prevalencia e incidencia son consideradas prioridades sanitarias (2)

Los factores predisponentes de estas anomalías, son diversa, desde características morfológicas, estructurales o funcionales. Debido a su etiología multifactorial, es complicado precisar los factores involucrados en su aparición, porque muchos de estos, pueden originar manifestaciones semejantes, aunque con variaciones, debido a que existe reacciones variadas en los sujetos ante la influencia estos factores de riesgo (2).

Se han identificado factores asociados de tipo congénitos, lesiones al nacer, enfermedad materna durante el embarazo, patologías de la garganta, nariz y oído, pérdida precoz de dientes temporales, hipotonía muscular, problemas psicológicos, lactancia o biberón prolongados, consistencia y composición de la dieta, traumatismos, hábitos deformantes entre otros factores involucrados (3).

En conclusión, las maloclusiones son alteraciones en el sistema estomatognático o irregularidades en el ordenamiento dental, huesos, tejidos blandos y articulaciones temporomandibulares (4).

La prevención de estas alteraciones es fundamental y está orientada a evitar las maloclusiones y presencia de anomalías, incluye el control de hábitos perjudiciales para el desarrollo estomatognático; la instalación de mantenedores de espacio en casos de extracción prematura de dientes deciduos; la extracción de supernumerarios o cualquier otro factor que altere el patrón eruptivo de las piezas permanentes y de otra medida de carácter mecánico o quirúrgico que prevenga la maloclusión (5).

El grado y tipo de alteración en la oclusión debido a la presencia de hábitos, es variada y depende de la intensidad, frecuencia y duración del hábito (tríada de Graber),(6-9) así como de aspectos biotipológicos del paciente.(7) No obstante son pocos los estudios que abordan la frecuencia y duración del hábito.(9-13)

Considerando este planteamiento, el objetivo del estudio fue comparar las anomalías dentofaciales según el sexo, en estudiantes de odontología de una universidad pública de Ica-Perú

\section{MATERIALES Y MÉTODOS}

Estudio de tipo observacional, transversal y descriptivo. La investigación se llevó a cabo durante el año 2019. El tamaño de la muestra fue de 60 estudiantes de odontología, seleccionados mediante muestreo probabilístico aleatorio simple, condicionado a cumplir los criterios de inclusión. Se incluyeron participantes de ambos sexos y que aceptaron participar del estudio. Se excluyeron los estudiantes que hayan recibido tratamiento ortodóncico y los que no aceptaron participar en la investigación.

Para identificar las anomalías dentofaciales, se utilizaron los criterios del Índice Estético Dental (IED) (14). Se registraron los siguientes apartados: Dientes incisivos, caninos y premolares perdidos; apiñamiento en los segmentos de los incisivos; separación de los segmentos de los incisivos; diastema de la línea media; máxima irregularidad anterior del maxilar; superposición anterior del maxilar; superposición anterior de la mandíbula; mordida abierta anterior vertical y relación molar anteroposterior. El análisis de los modelos de cada paciente fue registrado en una ficha de recolección de datos, todas las mediciones se hicieron con la sonda IPC.

Se utilizó la técnica de observación y se emplearon los modelos ortodóncicos de estudio de los alumnos de la Facultad de Odontología de la UNICA. Para conservar el anonimato de los participantes se colocó sobre el zócalo de los modelos el sexo del alumno a quien correspondía la impresión, luego se almacenaron cuidadosamente. Al recibir los modelos se descartaron aquellos que habían recibido tratamiento ortodóncico y los modelos que no cumplieron los criterios de inclusión. Se seleccionaron los modelos que cumplieron los criterios de inclusión y se enumeraron hasta completar 30 modelos de cada sexo.

Se preparó un libro de códigos, en base al instrumento y a la información obtenida, la cual fue incorporada en una base de datos. El análisis estadístico descriptivo incluyó medidas de frecuencia, porcentajes y promedios. Para evaluar diferencias la variable anomalías dentofaciales fue categorizada, como con anomalía y sin anomalía; se utilizó la prueba estadística Chi-cuadrado y se consideró estadísticamente significativos los resultados de las pruebas con $p<0,05$. Para el procesamiento de datos, se utilizó el paquete estadístico «Statistical Package for the Social Sciences» para Windows versión 25,0.

La investigación es absolutamente anónima solo se consideró el sexo del alumno en las impresiones para la confección de los modelos ortodóncicos de estudio; por tanto, no fue necesario tomar otras medidas para proteger la identidad, al trabajar sobre modelos, luego de obtener las impresiones no hubo ninguna intervención de contacto con los alumnos colaboradores en la investigación.

\section{RESULTADOS}

En cuanto al componente, incisivos, caninos y premolares perdidos, esta fue mayor en dientes superiores y en varones casi tres veces más que las mujeres. El apiñamiento incisivo la ocurrencia es similar tanto en varones como en mujeres. La separación entre los dientes del segmento incisivo es más frecuente entre las alumnas mujeres, tanto en un segmento como en ambos segmentos; en tanto, que la frecuencia de casos 
sin separación es homogénea tanto en varones como mujeres. Los diastemas de línea media solo se presentaron con una separación de $1 \mathrm{~mm}$ y es más frecuente en las mujeres. La máxima irregularidad maxilar más frecuente fue de $2 \mathrm{~mm}$, y en ambos sexos. La máxima irregularidad mandibular más recurrente fue de $2 \mathrm{~mm}$, tanto en varones como en mujeres. En el sexo masculino se presenta más frecuentemente la superposición anterior maxilar. La superposición anterior de la mandíbula tiene una frecuencia baja, siento esta mayor en el sexo masculino el valor de la superposición fue $-2 \mathrm{~mm}$, en todos los casos. Se encontró solo un caso de mordida abierta de -2 $\mathrm{mm}$, en el sexo masculino. La relación molar anteroposterior normal fue mayor en el sexo masculino; el desplazamiento de media cúspide, fue muy superior en el sexo femenino y el desplazamiento de una cúspide fue más frecuente en el sexo masculino. (Tabla 1)

Tabla $\mathbf{N}^{\circ}$ 1: Distribución de los componentes del Índice de Estética Dental, según el sexo de los estudiantes de odontología.

\begin{tabular}{|c|c|c|}
\hline \multirow{2}{*}{ Anomalías dentofaciales } & Varones & Mujeres \\
\hline & $\%$ & $\%$ \\
\hline \multicolumn{3}{|c|}{ Incisivos, caninos y premolares perdidos/segmentos } \\
\hline Superior & 310 & 13.3 \\
\hline Inferior & 0 & 0 \\
\hline Ambos & 0 & 0 \\
\hline S/ Faltantes & 2790 & 2996.3 \\
\hline \multicolumn{3}{|l|}{ Apiñamiento Incisivo } \\
\hline Sin apiñamiento & 516.7 & 516.7 \\
\hline Un segmento apiñado & 1343.3 & 1240.0 \\
\hline Dos segmentos apiñados & 1240.0 & 1343.3 \\
\hline \multicolumn{3}{|l|}{ Separación en el segmento Incisivo } \\
\hline Sin separación & 2893.3 & 2583.3 \\
\hline Un segmento con separación & 26.7 & 413.3 \\
\hline Dos segmentos con separación & $0 \quad 0$ & 13.3 \\
\hline \multicolumn{3}{|l|}{ Diastema } \\
\hline $1 \mathrm{~mm}$. & 13.3 & 26.7 \\
\hline S/ Diastema & 2996.7 & 2893.3 \\
\hline \multicolumn{3}{|l|}{ Máxima irregularidad maxilar } \\
\hline $0 \mathrm{~mm}$ & 1240.0 & 930.0 \\
\hline $1 \mathrm{~mm}$. & 310.0 & 723.3 \\
\hline $2 \mathrm{~mm}$. & 826.7 & 1137.7 \\
\hline $3 \mathrm{~mm}$. & 310.0 & 13.3 \\
\hline $4 \mathrm{~mm}$. & 13.3 & 13.3 \\
\hline $5 \mathrm{~mm}$. & 26.7 & 00.0 \\
\hline $6 \mathrm{~mm}$. & 00.0 & 13.3 \\
\hline $7 \mathrm{~mm}$ & 13.3 & 00.0 \\
\hline Máxima irregularidad mandibular & & \\
\hline $0 \mathrm{~mm}$ & 930.0 & 723.3 \\
\hline $1 \mathrm{~mm}$. & 620.0 & 723.3 \\
\hline $2 \mathrm{~mm}$. & 1240.0 & 1033.3 \\
\hline $3 \mathrm{~mm}$. & 13.3 & 620.0 \\
\hline $4 \mathrm{~mm}$. & 26.7 & 00.0 \\
\hline $5 \mathrm{~mm}$. & 00.0 & 00.0 \\
\hline $6 \mathrm{~mm}$. & 00.0 & 00.0 \\
\hline Superposición anterior del maxilar supe & & \\
\hline $0 \mathrm{~mm}$ & 310.0 & 26.7 \\
\hline $1 \mathrm{~mm}$. & 00.0 & 13.3 \\
\hline $2 \mathrm{~mm}$. & 413.3 & 513.3 \\
\hline $3 \mathrm{~mm}$. & 516.7 & 826.7 \\
\hline $4 \mathrm{~mm}$. & 413.3 & 826.7 \\
\hline $5 \mathrm{~mm}$. & 827.7 & 513.3 \\
\hline $6 \mathrm{~mm}$. & 413.3 & 13.3 \\
\hline $7 \mathrm{~mm}$ & 13.3 & 00.0 \\
\hline $8 \mathrm{~mm}$ & 13.3 & 00.0 \\
\hline Superposición anterior de la mandíbula & & \\
\hline S/ Superposición & 2893.3 & 2996.7 \\
\hline$-2 \mathrm{~mm}$. & 26.7 & 13.3 \\
\hline
\end{tabular}

\begin{tabular}{lcc} 
Mordida abierta anterior vertical & & \\
S/ M. Abierta & 2996.7 & 30100 \\
$-\quad 2$ mm. & 13.3 & 00.0 \\
Relación molar anteroposterior & & \\
Normal & 1963.3 & 1343.3 \\
Media cúspide & 826.7 & 1653.3 \\
Una Cúspide & 310.0 & 13.3 \\
\hline
\end{tabular}

$\mathrm{F}=$ Frecuencia absoluta; \%= Frecuencia relativa.

Respecto a la frecuencia de las anomalías dentofaciales la más recurrente fue el apiñamiento, máxima irregularidad mandibular, máxima irregularidad maxilar y la relación molar anteroposterior; las anomalías menos frecuentes son la mordida abierta, la superposición mandibular, el diastema y los dientes perdidos. Al evaluar diferencias entre la presencia de anomalías dentofaciales según el sexo, no se encontraron diferencias significativas $(p=0,464)$, dado que estas anomalías se presentan indistintamente según el sexo de los estudiantes de odontología.

Tabla $N^{\circ}$ 2: Frecuencia de anomalías dentofaciales, según el sexo de los estudiantes de odontología.

\begin{tabular}{lcccccc}
\multicolumn{1}{c}{ Anomalías Dentofaciales } & \multicolumn{2}{c}{ Varones } & \multicolumn{2}{c}{ Mujeres } & \multicolumn{2}{c}{ Total } \\
& F & \% & F & $\%$ & F & $\%$ \\
\hline Apiñamiento & 25 & 41.7 & 25 & 41.7 & 50 & 83.3 \\
Máxima I. Mandib & 21 & 35.0 & 23 & 38.3 & 44 & 73.3 \\
Máxima I. Maxilar & 18 & 30.0 & 21 & 35.0 & 39 & 65.0 \\
Relación molar & 11 & 18.3 & 17 & 28.3 & 28 & 46.7 \\
Superposición maxilar & 14 & 23.3 & 6 & 10.0 & 20 & 33.3 \\
Separación S.I & 2 & 3.3 & 5 & 8.3 & 7 & 11.7 \\
Dientes perdidos & 3 & 5.0 & 1 & 1.7 & 4 & 6.7 \\
Diastema & 1 & 1.7 & 2 & 3.3 & 3 & 5.0 \\
Superposición A mandibular & 2 & 3.3 & 1 & 1.7 & 3 & 5.0 \\
Mordida abierta & 1 & 1.7 & 0 & 0.0 & 1 & 1.7 \\
\hline
\end{tabular}

\section{DISCUSIÓN}

Se encontró en la muestra estudiada, respecto a las anomalías dentofaciales, que los dientes perdidos se presentan exclusivamente en la arcada superior y con mayor frecuencia en el sexo masculino, el $96 \%$ no presentó dientes perdidos. Por su parte Peláez (15), encontró en una muestra conformada por 154 pacientes que concurrieron para su atención integral al Hospital Universitario Odontológico de la Facultad de Odontología de la Universidad Nacional de Nordeste (UNNE) que un $48 \%$ presentó pérdida de piezas dentarias.

La presencia del índice de apiñamiento fue muy elevado en comparación con los promedios conocidos; Menéndez (16), reportó un apiñamiento de solo $28 \%$ y Peláez (15), $44 \%$ de apiñamiento en ambos segmentos. Para este trabajo tenemos un total de $83.3 \%$ para ambos segmentos. El apiñamiento se presenta casi con la misma frecuencia tanto en un segmento o en dos segmentos y por igual en ambos sexos.

Respecto a la separación en los segmentos incisivos, dos de cada diez estudiantes presenta esta anomalía. El indicador diastema (separación en la línea media superior) se presentó un caso en el sexo masculino y dos en el sexo femenino. La máxima irregularidad anterior del maxilar fue $7 \mathrm{~mm}$ y la máxima irregularidad anterior de la mandíbula $4 \mathrm{~mm}$. El 16,6 \% superó la superposición maxilar superior normal en el sexo femenino, pero en el sexo masculino la frecuencia de esta anomalía es muy superior, llegando al 47,6 \%. Por su parte, Menéndez (16), 
encontró una superposición en el 50\% mayor de $6 \mathrm{~mm}$, en el presente estudio no se encontró ningún caso de superposición mayor de $6 \mathrm{~mm}$, en el sexo femenino. En el sexo masculino hallamos una superposición mandibular de $-2 \mathrm{~mm}$, en el $6,7 \%$ y en el sexo femenino 3,3\%; similar a Menéndez (16), para quién esta anomalía estuvo presente en el $4 \%$.

La mordida abierta se presentó en el 3,3 \% de sexo masculino con un valor de $-2 \mathrm{~mm}$, en el sexo femenino no se encontró ningún caso, difiriendo Menéndez (16), quién reporta 9\% de mordida abierta en el sexo femenino.

Finalmente la relación molar anteroposterior se presentó normal mayormente en el sexo masculino, el desplazamiento de media cúspide, fue muy superior el sexo femenino, por su parte Peláez (15), revela $49 \%$ en ésta anomalía, muy similar a los hallazgos de esta investigación.

Respecto a la prevalencia de las maloclusiones, esta fue alta en la muestra analizada, diversos estudios reportan resultados similares (17-20)

En cuanto a las limitaciones del estudio podemos declarar el tamaño de la muestra; tal vez puede ser considerada no representativa de la población, por tanto sugerimos replicar el estudio con muestras más grandes y en poblaciones distintas. El nivel de investigación es descriptivo-relacional, no pudiendo establecer una relación de causalidad entre las variables evaluadas; a pesar de ello, caracterizar y comparar las variables de estudio es importante, porque nos permite identificar y atender necesidades específicas de salud bucal, en la población de estudio.

Se concluye que no existen diferencias significativas entre la presentación de anomalías dentofaciales, según el sexo en la muestra estudiada; no obstante, se describen hallazgos relevantes respecto a la frecuencia de estas anomalías, a tener en cuenta en los planes a atención integral de los estudiantes de odontología. 


\section{REFERENCIAS BIBLIOGRÁFICAS}

1. Fernández Martínez J, Mora Pérez C, López Fernández R. Intervención educativa y terapéutica en niños portadores de hábitos bucales deformantes. Revista Electrónica de las Ciencias Médicas en Cienfuegos. 2009; 7 (1): 28-48.

2. Aznar Martín T, Galán González AF, Martín Castro IM, Domínguez Reyes A. Dental Arch Diameters and relationship to oral habits. Angle Orthodontist. 2005;76(3):34-8.

3. Paredes Gallardo V, Paredes Censillo C. Prevalencia de los hábitos bucales y alteraciones dentarias en escolares valencianos. An Pediatr. 2005;62(3):261-5.

4. León Caballero KM, Maya Hernández B, Vega Galindo M, Mora Pérez C. Factores de riesgo asociados con anomalías de oclusión en dentición temporal. Área III. Rev Cubana Estomatol. 2007;44(4):4. Disponible en: http://bvs.sld.cu/revistas/est/vol44- 04-07 /est03407.htm

5. Van Maes HJ, Stökli P. Atlas de Odontología Pediátrica. Masson, Barcelona: 2002

6. León AJ, Carvajal RY, Pérez HN, Rodríguez MO. Hábitos bucales deformantes y su posible influencia sobre el plano poslácteo en niños con dentición temporal. Mediciego [Revista en Internet]. 2014 [citado 22 Ago 2016];20(Supl 1):[aprox. 5 p.]. Disponible en: http://bvs.sld.cu/revistas/mciego/vol20_Supl\%201_14/articu los/T8.html

7. Franco VV, Gorritxo GB. Hábito de succión del chupete y alteraciones dentarias asociadas. Importancia del diagnóstico precoz. An Pediatr (Barc). [Revista en Internet]. 2012 May [citado 15 Ago 2021];77(6):374-80. Disponible en: http://www.analesdepediatria.org/es/habito-succion-del-ch upetealteraciones/articulos/S1695403312001518/

8. Franco Varas V, Gorritxo Gil B, García IF. Prevalencia de hábitos orales infantiles y su influencia en la dentición temporal. Rev Pediatr Aten Primaria. 2012 [citado 15 Ago 2021];14(53):13-20. Disponible en: http://scielo.isciii.es/scielo.php?script=sci_arttext\&pid $=\mathrm{S} 113$ 9-76322012000100002

9. Garbin CAS, Garbin AJl, Martins RJ, de Souza NP, Moimaz SAS. Prevalência de hábitos de sucção não nutritivos em pré-escolares e a percepção dos pais sobre sua relação com maloclusões. Ciênc. Saúde Coletiva [Revista en Internet]. 2014 Feb. [citado 15 Ago 2021]; 19(2):553-8. Disponible en: http://www.scielo.br/pdf/csc/v19n2/1413-8123-csc-19-02-0 0553.pdf

10. Sousa RV, Viera de Andrade RA, Ribeiro GLA, Firmino RT, Martins CC, GranvilleGarcia AF, Paiva S. Prevalence and associated factors for the development of anterior open bite and posterior crossbite in the primary dentition. Braz Dent J [Internet]. 2014 [cited 2021 Ago 15];25(4):336-42. Available from:

http://www.scielo.br/scielo.php?script=sci_arttext\&pid=S01 03-64402014000400336\&lng=en

11. Moimaz SAS, Garbin AJI, Lima AMC, Lolli LF, Saliba O, Garbin CAS. Longitudinal study of habits leading to malocclusion development in childhood. BMC Oral Health [Internet]. 2014 [cited 2021 Ago 15];14:96. Available from: http://www.ncbi.nlm.nih.gov/pmc/articles/PMC4126276/pdf /1472-6831-14-96.pdf

12. Nihi VSC, Maciel SM, Jarrus ME, Nihi FM, de Salles CLF, Pascotto $\mathrm{RC}$, et al. Pacifiersucking habit duration and frequency on occlusal and myofunctional alterations in preschool children. Braz Oral Res [Internet]. 2015 [cited 2021 Ago 14];29(1):1-7. Available from: http://www.scielo.br/scielo.php?script=sci_pdf\&pid=S1806$83242015000100211 \& \mathrm{lng}=$ en\&nrm=iso\&tlng=en
13. Ize-lyamu IN, Isiekwe MC. Prevalence and factors associated with anterior open bite in 2 to 5 year old children in Benin city, Nigeria. Afr Health Sci [Internet] 2012 Dec. [cited 2021 Ago 14];12(4):446-51. Available from: http://www.ncbi.nlm.nih.gov/pmc/articles/PMC3598284/pdf /AFHS1204-0446.pdf

14. Jenny J, Cons NC. Guide Lines for using the DAI. A supplement ro DAl, the Dental Aesthetic Index. Iowa City; University of lowa; 1988 .p.7.

15. Peláez A. Prevalencia, severidad de maloclusion y necesidad de tratamiento ortodóncico según el índice estético dental revista Facultad de Odontologia ISSN $\mathrm{N}^{\circ}$ 1668-7280.2016:6(1):73-7.

16. Menéndez L. Estudio comparativo de tres índices de maloclusiones. OMS, AIO, AGUILA, en un grupo de escolares de 11 a 16 años de edad de sexo femenino en la ciudad de Lima. Odontología Sanmarquina 1(4): Julio-diciembre 1999.

17. Pérez M. Necesidad de Tratamienro Ortodóncico según el Índice de Estetica Dental en adolescentes de 12 años, Chile. Revista Faculdad Odontología Universidad de Antoquia. 2014; 26(1).

18. Gutiérrez M. Necesidad de tratamiento ortodóntico utilizando el Índice Estética Dental (DAI) en una población de Guadalajara, Jalisco, México. Univeridad Nacional de Colombia [Internet]. 2016; 6(1). Disponible en: https://revistas.unal.edu.co/index.php/actaodontocol/article /view/58851

19. Navarro J. Percepción de la necesidad de tratamiento ortodóncico por parte de adolescentes y sus padres. 2018;22(1). Disponible en: http://scielo.sld.cu/scielo.php?script=sci_arttext\&pid=S1029 -30192018000100004

20. Oliveira J. Índice de estética dental en niños en la consulta de Odontopediatría. Polo del conocimiento. 2018;3(5). 